LAWRENCE LIVERMORE N A TION AL LABORATORY
Interface Structure and Atomic Bonding Characteristics in Silicon Nitride Ceramics

A. Ziegler, J. C. Idrobo, M. K. Cinibulk, C. Kisielowski, N. D. Browning, R. O. Ritchie

October 12, 2004

Science 
This document was prepared as an account of work sponsored by an agency of the United States Government. Neither the United States Government nor the University of California nor any of their employees, makes any warranty, express or implied, or assumes any legal liability or responsibility for the accuracy, completeness, or usefulness of any information, apparatus, product, or process disclosed, or represents that its use would not infringe privately owned rights. Reference herein to any specific commercial product, process, or service by trade name, trademark, manufacturer, or otherwise, does not necessarily constitute or imply its endorsement, recommendation, or favoring by the United States Government or the University of California. The views and opinions of authors expressed herein do not necessarily state or reflect those of the United States Government or the University of California, and shall not be used for advertising or product endorsement purposes. 


\title{
Interface Structure and Atomic Bonding Characteristics in Silicon Nitride Ceramics
}

\author{
A. Ziegler ${ }^{1,2}$, J. C. Idrobo ${ }^{3}$, M. K. Cinibulk ${ }^{4}$, C. Kisielowski ${ }^{5}$, \\ N. D. Browning ${ }^{3,5}$ and R. O. Ritchie ${ }^{1,6}$
}

${ }^{1}$ Materials Sciences Division, Lawrence Berkeley National Laboratory, Berkeley, CA 94720, USA

${ }^{2}$ Materials Science and Technology Division, Chemistry and Materials Science Directorate, Lawrence Livermore National Laboratory, Livermore, CA 94551, USA

${ }^{3}$ Physics Department, University of California, Davis, CA 95616, USA

${ }^{4}$ Materials and Manufacturing Directorate, Air Force Research Laboratory, Wright-Patterson Air Force Base, OH 45433, USA

${ }^{5}$ National Center for Electron Microscopy, Lawrence Berkeley National Laboratory, Berkeley, CA 94720, USA

${ }^{6}$ Department of Materials Science and Engineering, University of California, Berkeley, CA 94720, USA

submitted to Science

August 2004

Rev. October 2004

Work supported by the Director, Office of Science, Office of Basic Energy Sciences, Division of Materials Sciences and Engineering of the U.S. Department of Energy Department of Energy under Contract No. W-7405-Eng-48, DE-AC03-76SF00098 and FG02-03ER-46057.

This work was performed under the auspices of the U.S. Department of Energy by the University of California, Lawrence Livermore National Laboratory under Contract No. W-7405-Eng-48. 


\title{
Interface Structure and Atomic Bonding Characteristics in Silicon Nitride Ceramics
}

\author{
A. Ziegler ${ }^{1,2}$, J. C. Idrobo ${ }^{3}$, M. K. Cinibulk ${ }^{4}$, C. Kisielowski ${ }^{5}$, \\ N. D. Browning ${ }^{3,5}$ and R. O. Ritchie ${ }^{1,6}$ \\ ${ }^{1}$ Materials Sciences Division, Lawrence Berkeley National Laboratory, Berkeley, CA \\ 94720, USA \\ ${ }^{2}$ Materials Science and Technology Division, Chemistry and Materials Science \\ Directorate, Lawrence Livermore National Laboratory, Livermore, CA 94551, USA \\ ${ }^{3}$ Physics Department, University of California, Davis, CA 95616, USA \\ ${ }^{4}$ Materials and Manufacturing Directorate, Air Force Research Laboratory, Wright- \\ Patterson Air Force Base, OH 45433, USA \\ ${ }^{5}$ National Center for Electron Microscopy, Lawrence Berkeley National Laboratory, \\ Berkeley, CA 94720, USA \\ ${ }^{6}$ Department of Materials Science and Engineering, University of California, Berkeley, \\ CA 94720, USA
}

\section{One-sentence summary}

This investigation examines the interface atomic structure and bonding characteristics in an advanced ceramic, obtaining new and unique experimental information that will help to understand and improve the properties of ceramics.

\section{$\underline{\text { Abstract }}$}

Unique direct atomic resolution images have been obtained that illustrate how a range of rare-earth atoms bond to the interface between the intergranular phase and the matrix grains in an advanced silicon nitride ceramic. It has been found that each rare-earth atom bonds to the interface at a different location, depending on atom size, electronic configuration and the presence of oxygen at the interface. This is the key factor to understanding the origin of the mechanical properties in these ceramics and will enable precise tailoring in the future to critically improve the materials performance in wide-ranging applications.

Bulk silicon nitride $\left(\mathrm{Si}_{3} \mathrm{~N}_{4}\right)$ ceramics have been investigated extensively over the last two decades, largely because their mechanical and physical properties are relevant 
for many high-temperature applications, including high strength, high decomposition temperature $\left(1900^{\circ} \mathrm{C}\right)$, good oxidation resistance, low coefficient of friction, negligible creep, good thermal shock properties, and good resistance to corrosive environments. In addition, thin silicon nitride films and coatings have been studied in order to understand electrical and thermal conductivity properties. All of these properties, including the processing and sintering behavior, rely on the specific crystal structure, the local chemistry, and the local bonding at the interfaces.

The widespread use of these ceramics as structural components is currently limited by their brittleness that limits reliability. Specifically, the toughness is too low and the sensitivity to flaws and cracks correspondingly too high, resulting in poor damage tolerance. Such properties can be improved by microstructural and compositional design. Indeed, the relationship between microstructure, intergranular phases and mechanical properties in ceramics is widely acknowledged $(1,2)$. Silicon nitride ceramic microstructures consist of elongated matrix-grains that are randomly oriented, interlocked, and interspersed with a secondary phase (3-8). This intergranular phase in these materials is the key microstructural element that effectively controls most of the material's characteristics, most notably the mechanical properties, and is dependent on the local chemical composition, atomic structure and bonding characteristics (9-13).

Previous investigations have shown empirically that the material's strength and toughness are markedly affected by the intergranular phase chemistry. An understanding of how this phase governs the macroscopic mechanical properties is limited, because the amorphous morphology of the phase and its small dimension make analysis difficult. 
Recent breakthroughs of scanning transmission electron microscopes (STEM) and associated chemical analysis (14-18) permit probing the local atomic structure and bonding characteristics with a resolution close to 1 Ångstrøm.

We examine the immediate interface between matrix grains and the amorphous intergranular film, extracting unique structural and atomic bonding information. In particular, we relate the structure of the interface to the atomic size and electronic structure of the rare-earth elements in the intergranular film. The oxides of rare-earth elements are a very common type of sintering additive in $\mathrm{Si}_{3} \mathrm{~N}_{4}$ as it has been shown empirically that such additions improve the mechanical properties $(12,13)$. We investigate the interface between the intergranular phase and the $\mathrm{Si}_{3} \mathrm{~N}_{4}$ matrix using highangle annular dark-field STEM (HAADF-STEM) for imaging and electron-energy-lossspectroscopy (EELS) (14) for chemical analyses. Experimental electron-energy-lossspectra (EELS) and Z-contrast images (15) were obtained with a monochromated FEI Tecnai F20 scanning-transmission-electron-microscope (STEM), operating at $200 \mathrm{kV}$. The lens conditions in the microscope were defined for imaging and spectroscopy with a probe size of $0.14 \mathrm{~nm}$. The inner semi-angle of the annular dark-field detector (ADF) was chosen to be $74 \mathrm{mrad}$ and $110 \mathrm{mrad}$. All images were acquired with an objective semiangle of $13.5 \mathrm{mrad}$. These experimental conditions are enough to minimize the effects of strain fields on the Z-contrast image (16). In this experimental condition, the incoherent high-angle annular dark field or "Z-contrast" image allows the structure of the grains to be directly observed, and the image can also be used to position the electron probe for EELS (14). 
Previous investigations of these interfaces have focused on two common additives, $\mathrm{Y}_{2} \mathrm{O}_{3}$ and $\mathrm{La}_{2} \mathrm{O}_{3}(7,8)$, showing primarily that there is $\mathrm{Y}$ and La segregation in the intergranular phase. Furthermore, the most recent study (8) uses the HAADF-STEM imaging technique described above and first-principles calculations to show that there is variability in this segregation at the interface between the matrix grains and intergranular phase. However, the techniques employed did not give information about the electronic configuration or the specific atomic location for the additives at the interface. Our objective is the identification of bonding sites for a larger selection of rare-earth elements of the following oxide sintering additives, $\mathrm{La}_{2} \mathrm{O}_{3}, \mathrm{Sm}_{2} \mathrm{O}_{3}, \mathrm{Er}_{2} \mathrm{O}_{3}, \mathrm{Yb}_{2} \mathrm{O}_{3}$ and $\mathrm{Lu}_{2} \mathrm{O}_{3}$, and to extend previous investigations by analyzing their electronic configuration and determining the atomic locations of the additives at the interface with the intergranular phase. We take advantage of the "chemically-sharp" interface between the intergranular phase and matrix grains caused by a miscibility gap between the $\mathrm{Si}_{3} \mathrm{~N}_{4}$ crystal and any of the rare-earth atoms, i.e., these atoms do not substitute for $\mathrm{Si}_{3} \mathrm{~N}_{4}$ host atoms in their atomic positions nor do they sit at interstitial sites in the $\mathrm{Si}_{3} \mathrm{~N}_{4}$ crystal structure.

The HAADF-STEM images in Fig. 1 depict an interface between the intergranular phase and a $\mathrm{Si}_{3} \mathrm{~N}_{4}$ matrix grain for each of the five sintering additive types. The images confirm that the $\mathrm{Si}_{3} \mathrm{~N}_{4}$ crystal structure ends at the interface with the intergranular phase, namely with open hexagonal rings (7). The attachment of heavy atoms can be proven by the Z-contrast in the images that makes heavier atoms appear brighter. Concomitant large-area EELS measurements performed on these interfaces confirm that the heavy 
elements indeed segregate to the intergranular phase. Hence, the bright spots seen in the images are indeed related to the heavy atoms and their atomic positions. Accordingly, the atomic attachment at such an interface is different for each of the rare-earth elements ( $\mathrm{La}$, $\mathrm{Sm}, \mathrm{Er}, \mathrm{Yb}$ and $\mathrm{Lu}$ ) examined here. The STEM images of the sample containing La show no specific or periodic connection occurring between the $\mathrm{Si}_{3} \mathrm{~N}_{4}$ matrix grain and the $\mathrm{La}$ atoms. The La atoms are present in the intergranular phase, as reported previously (8), yet they do not arrange into atomic columns that would increase contrast. Additionally, no lateral atomic ordering can be observed at this magnification. Previous investigations have shown how the La atom distribution along the intergranular phase fluctuates, with locations of high and low La atom concentration (8). The distance between the areas of high $\mathrm{La}$ concentration are rather far apart and as such cannot be associated with a particular atomic site along the interface. Concomitant theoretical calculations attempting to determine specific atomic sites are of help; however, in agreement with the present results, a periodic atomic site for La atom attachment cannot be discerned unambiguously from the unaided STEM images.

In contrast, the images of the other four compositions containing $\mathrm{Sm}, \mathrm{Er}, \mathrm{Yb}$, and $\mathrm{Lu}$, exhibit very different features. Here, the bright spots that appear at the interface in the amorphous intergranular phase, are attributed to columns of $\mathrm{Sm}, \mathrm{Er}, \mathrm{Yb}$ and $\mathrm{Lu}$ atoms, respectively, due to their high contrast. These images clearly show that the atomic bonding of those atoms along the $\mathrm{Si}_{3} \mathrm{~N}_{4}$ prismatic plane is periodic and occurs at very specific atomic sites, $\mathrm{A}$ and $\mathrm{B}$, as indicated in Fig. 1. Sm atoms bond in single-atom configuration to both positions, A and B. This changes with the slightly heavier, but 
smaller, atoms Er, $\mathrm{Yt}$ and $\mathrm{Lu}$, as they bond in pairs at position A with the pair-axis oriented parallel to the prismatic plane. It cannot be determined to what degree the axis is also parallel to the image plane, but observing how close the bright spots are and considering the atomic size of these elements, it follows that the atom-pair axes are inclined and are seen in projection. The atom-pairs of $\mathrm{Lu}, \mathrm{Yb}$ and $\mathrm{Er}$ appear to be separated differently; Lu: $1.43 \pm 0.07 \AA, \quad \mathrm{Yb}: 1.46 \pm 0.05 \AA$ and $\mathrm{Er}: 1.48 \pm 0.04 \AA$. This growing pair separation can be related to the increase of the valence shell radius from $\mathrm{Lu}$ to Er (Fig. 1), which may also be the cause for occasional pair-splitting that can be observed in the images, i.e., some atom-pairs are separated more than others. For Er and $\mathrm{Yb}$, this results in an occasional larger atom-pair separation of $2.06 \pm 0.03 \AA$ and $2.15 \pm 0.1 \AA$, respectively. Upon close examination of these split atom pairs, there is indication that beyond the first row of attached atoms there is a second set of rare-earth atoms, reaching even farther into the amorphous intergranular phase - possibly a partial ordering imposed by the $\mathrm{Si}_{3} \mathrm{~N}_{4}$ crystal structure onto the amorphous intergranular phase. However, precise atomic positions beyond the first layer of atomic columns cannot be discerned at this point. These observations demonstrate that the bonding of the rare-earth elements to the interface is controlled by atomic size. The small atoms attach in pairs at specific and periodic sites along the interface. With increasing atom size the pairseparation increases to the point where larger atoms, like in the case of Sm, are not able to bond in pairs at position $\mathrm{A}$ and have to adapt a new single-atom, periodic bonding configuration. Consequently, one of them has to move to position B. With a further increase in atom size, i.e., La atoms, the attachment to the interface changes again, this 
time forfeiting all periodicity and site-specific atomic bonding. The question remains, however, why the small atoms preferably bond to position A and not B.

The initial EELS measurements, performed by area-scanning a rather large region of the intergranular films indicated atom segregation to the interface. More precise EELS measurements attempting to identify specific atomic bonding configurations were performed on the Sm-containing sample with a $1.4 \AA$ probe size, since this sample was the least electron-beam sensitive and it showed rare-earth atom attachment to positions $\mathrm{A}$ and B. Such a small probe size allows positioning of the electron beam over a single atomcolumn analyzing the very local bonding characteristics (14). The results, presented in Fig. 2, show the electron loss spectra for the $\mathrm{Si} L_{23}$ and the $\mathrm{Sm} N_{45}$ edges, respectively, measured on both identified atomic positions (A and B) along the interface. There is no difference between the spectra at the $\mathrm{Sm} N_{45}$ edge, suggesting that the atomic environment and bonding characteristics are the same for the Sm atoms at both locations, i.e., Sm exists in the same formal valence state in each location. The difference in the electron loss spectra from position $\mathrm{A}$ and $\mathrm{B}$ is at the $\mathrm{Si} L_{23}$ edge. A double peak $(102 \mathrm{eV}$ and $103.5 \mathrm{eV}$ ) appears in the spectrum taken from atom position A. The first peak can be identified with a Si-N bond while the second peak is associated with a $\mathrm{Si}-\mathrm{O}$ bond because its position in the spectrum closely coincides with measurements taken on $\mathrm{SiO}_{2}$ (19). This indicates that the atomic environment around the terminating $\mathrm{Si}$ atoms on the $\mathrm{Si}_{3} \mathrm{~N}_{4}$ prism plane that surround position A is most likely an oxygen atom. In contrast, the atomic environment of the Si atoms on the prism plane that surround position B appears to have no oxygen atoms involved, only nitrogen. 
The observation that the $\mathrm{Er}, \mathrm{Yb}$ and $\mathrm{Lu}$ atom-pairs preferably bond to position $\mathrm{A}$ suggests that the interaction of the rare-earth atom with the oxygen at site A plays a critical role in controlling atom attachment at the interface. Thus a combination of both factors, i.e., the difference in atomic size and presence of oxygen govern atom attachment and site selection along the interface. Note that this results in different atom concentrations at the individual positions $\mathrm{A}$ and $\mathrm{B}$. The rare-earth atom concentration is high for position A and comparably low at position B. The interaction of electronic configuration, atom size, and position of the rare-earth atoms on the prismatic plane is currently being investigated by atomic-structure calculations of this interface.

This information about the specific atomic structure and bonding characteristics in advanced ceramics has been missing for many years and should now aid the development of improved ceramics in many ways. Most importantly, it will assist in understanding how ceramic microstructures evolve during fabrication, especially how grain growth and microstructural evolution is affected by different sintering additives at the atomic level. Control over evolution and the ability to precisely tailor ceramic microstructures is critical in improving mechanical properties of ceramics, which invariably limits their use. From previous empirical investigations $(1,2,9,10,12,13)$ it is known how ceramics fracture is highly sensitive to the intergranular phase chemistry, and therefore its atomic structure and bonding characteristics. The new information concerning precise atom location from the present study should also prove invaluable for atomic-structure calculations. Such computational methods (20-26) seek to calculate individual atomic 
bonding strengths in order to explain the particular fracture mechanisms that occur along and across the intergranular phase. However, lacking essential experimental information about the atomic structure and bonding characteristics, such calculations have been incomplete.

Thus in summary, this study involving high-resolution STEM imaging and EELS measurements along the interface between $\mathrm{Si}_{3} \mathrm{~N}_{4}$ matrix grains and the intergranular phase in silicon nitride ceramics doped with $\mathrm{La}, \mathrm{Sm}, \mathrm{Er}, \mathrm{Yb}$ and $\mathrm{Lu}$, has shown at the atomistic scale how each rare-earth element attaches to the interface differently, depending on atomic size, electronic configuration and presence of oxygen atoms along the interface. 


\section{References}

1. Wiederhorn. S., Annual Review of Materials Science 14373 (1984).

2. $\quad$ Becher, P. F., Hwang, S. L., Hsueh, C. H., MRS Bull. 2023 (1995).

3. Clarke, D.R., J. Am. Ceram. Soc. 7015 (1987).

4. Kleebe, H.J., Cinibulk, M.K., Cannon, R.M., Rühle, M., J. Am. Ceram. Soc. 76 1969 (1993).

5. Wang, C. M, Pan, X., Hoffmann, M. J. Cannon, R. M., Rühle, M., J. Am. Ceram. Soc. 79788 (1996).

6. Tanaka, I. et al., J. Am. Ceram. Soc. 77911 (1994).

7. Ziegler, A., Kisielowski, C., Hoffmann, M. J., Ritchie, R. O., J. Am. Ceram. Soc. 861777 (2003).

8. Shibata, N. et al., Nature 428730 (2004).

9. Sun, E. Y. et al., J. Am. Ceram. Soc. 81, 2831 (1998).

10. Lange, F. F., Davis, B. I., Metcalf, M. G., J. Mat. Sci. 181497 (1983).

11. Satet, R. L, Hoffmann, M. J., J. Eur. Ceram. Soc. 243437 (2004).

12. Cinibulk, M. K. Thomas, G., Johnson, S. M., J. Am. Ceram. Soc. 752050 (1992).

13. Sanders, W. A., Mieskowski, D. M., J. Am. Ceram. Soc., 64304 (1985).

14. Browning, N. D., Chisholm, M. F., Pennycook, S. J., Nature 366143 (1993).

15. Nellist P.D., Pennycook S. J., Ultramicroscopy 78111 (1999).

16. Pennycook, S. J., Jesson, D. E., Phys. Rev. Lett. 64939 (1990).

17. James, E. M., Browning, N. D., Ultramicroscopy 78125 (1999).

18. Voyles, P. M. et al., Nature 416826 (2002). 
19. Gu, H., Cannon, R. M., Rühle, M., J. Mat. Res. 13376 (1998).

20. Nakayasu, T. et al., J. Am. Ceram. Soc. 81565 (1998).

21. Yoshiya, M., Tatsumi, K., Tanaka, I., Adachi. H., J. Am. Ceram. Soc. 85109 (2002).

22. Benco, L'., Surface Science 327274 (1995).

23. Benco, L'., Hafner, J, Lencés, Z., Sajgalík, P., J. Am. Ceram. Soc. 861162 (2003).

24. Tanaka, I. et al., Acta Met. Mat. 401995 (1992).

25. Ching, W.-Y., Huang. M. Z., Mo, S. D., J. Am. Ceram. Soc. 83780 (2000).

26. Garofalini, S. H., Luo, W., J. Am. Ceram. Soc. 861741 (2003). 


\section{Acknowledgements}

The authors would like to thank Prof. M. J. Hoffmann and Dr. R. L. Satet from IKM

Karlsruhe, Germany, for providing some of the ceramic material for this investigation. This work was supported by the Director, Office of Science, Office of Basic Energy Sciences, Division of Materials Sciences and Engineering, of the U.S. Department of Energy under Contract No. DE-AC03-76SF00098 and FG02-03ER-46057. 


\section{LIST OF FIGURE CAPTIONS}

Fig. 1. In these STEM images of $\mathrm{La}, \mathrm{Sm}, \mathrm{Er}, \mathrm{Yb}$, and $\mathrm{Lu}$-doped $\mathrm{Si}_{3} \mathrm{~N}_{4}$, the matrix grain is oriented along the [0001] zone axis such that the open $\mathrm{Si}_{3} \mathrm{~N}_{4}$ crystal structure is clearly visible at the atomic level and its prismatic plane faces the amorphous intergranular phase. The images show how the $\mathrm{Si}_{3} \mathrm{~N}_{4}$ crystal structure ends at the interface with the intergranular phase, namely with open hexagonal rings (7). For a material with strong covalent bonding this seems unusual, but it indicates that the prismatic plane may contain dangling bonds that can attract atoms from the intergranular phase. The attachment of heavy atoms, here $\mathrm{La}, \mathrm{Sm}, \mathrm{Er}, \mathrm{Yb}$ and $\mathrm{Lu}$, can be proven by the Z-contrast in the images that makes heavier atoms appear brighter. Furthermore, the atoms attach in the form of atomic columns oriented normal to the image plane, which contributes to their strong Zcontrast. Two distinct atom positions, $\mathrm{A}$ and $\mathrm{B}$, can be identified along the interface.

Fig. 2. Precise EELS measurements to identify the individual bonding characteristics of each of the rare-earth atomic locations were difficult to perform, primarily because the material is electron-beam sensitive and atoms were knocked-out of their specific atomic position before measurements could be done. Nevertheless, the $\mathrm{Si}_{3} \mathrm{~N}_{4}$ containing $\mathrm{Sm}$ was the least beam sensitive and permitted this critical EELS measurement. The results for (A) the $\mathrm{Si} L_{23}$ and (B) the $\mathrm{Sm} N_{45}$ edges identify the individual bonding characteristics at positions A and $\mathrm{B}$. The Si $L_{23}$ edge shows a double peak at position A but not at position B. The first of the double peak can be identified with a Si-N bond because it coincides 
with measurements taken on the $\mathrm{Si}_{3} \mathrm{~N}_{4}$ crystal structure. Identification of the second peak is achieved by comparing the results to reference spectra obtained from $\mathrm{SiO}_{2}$ and $\mathrm{Si}_{2} \mathrm{~N}_{2} \mathrm{O}$. Accordingly, the peak in question can be associated with a Si-O bond. Regarding the electronic environment for the $\mathrm{Sm}$ atoms at positions $\mathrm{A}$ and $\mathrm{B}$, there is no difference between the spectra at the $\mathrm{Sm} N_{45}$ edge. 


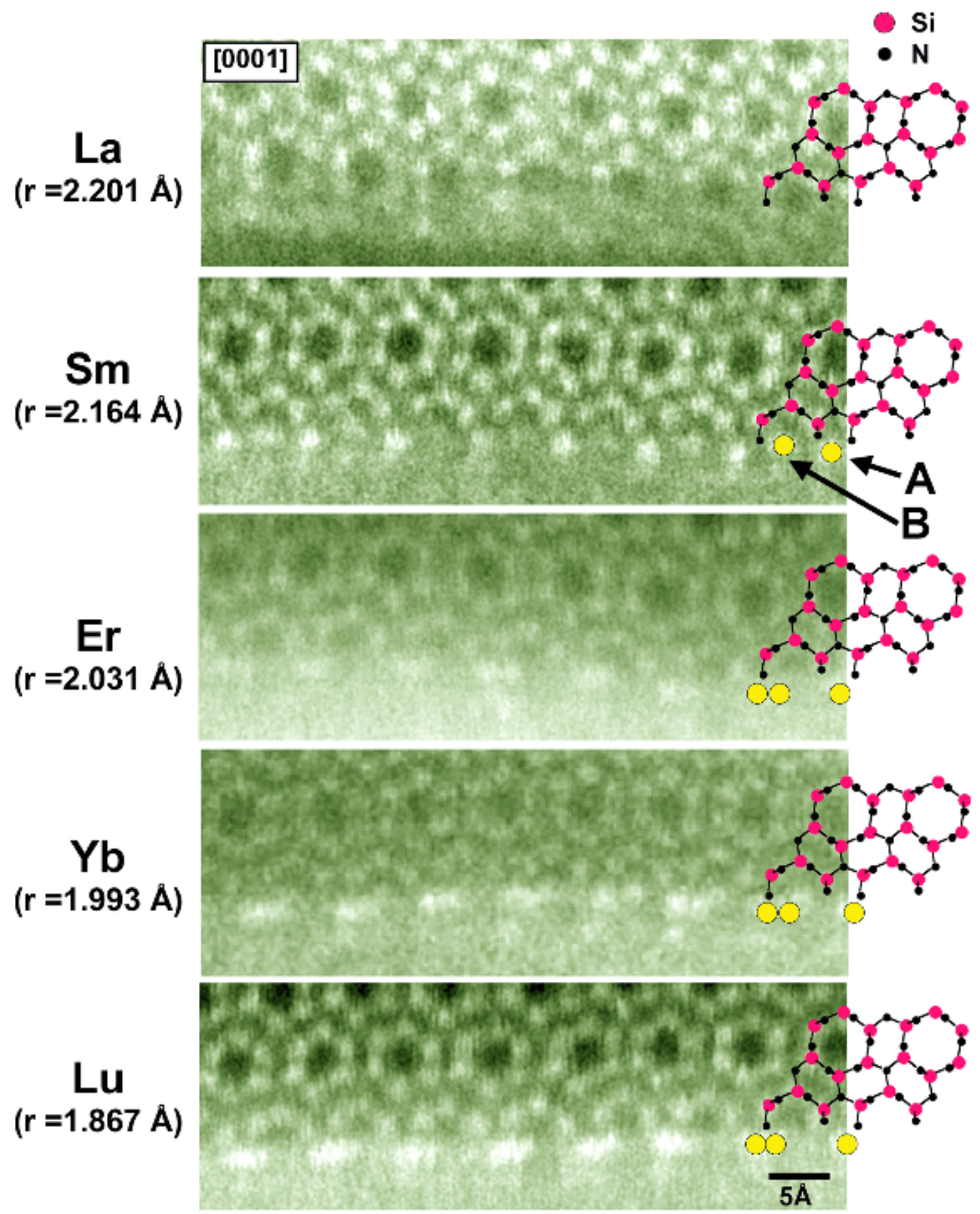

Fig. 1 

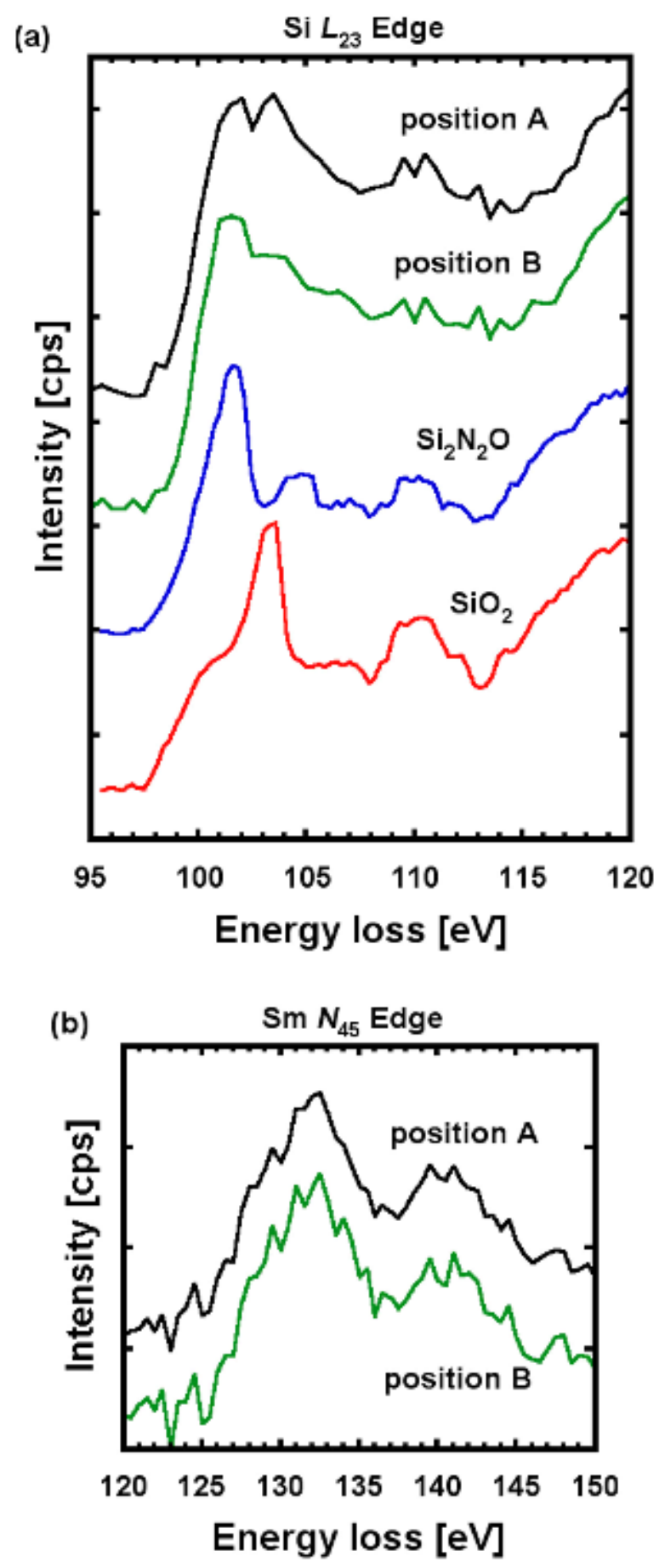

Fig. 2 